Relations industrielles

Industrial Relations

\title{
Les conflits de droit dans les rapports collectifs du travail. Par Marie-Louis Beaulieu. Québec: Les Presses Universitaires Laval, 1955. 540pp. En vente chez l'auteur, 945 avenue Bégin, Quélbec.
}

\section{Roger Chartier}

Volume 13, numéro 3, juillet 1958

URI : https://id.erudit.org/iderudit/1022432ar

DOI : https://doi.org/10.7202/1022432ar

Aller au sommaire du numéro

\section{Éditeur(s)}

Département des relations industrielles de l'Université Laval

\section{ISSN}

0034-379X (imprimé)

1703-8138 (numérique)

Découvrir la revue

Citer ce compte rendu

Chartier, R. (1958). Compte rendu de [Les conflits de droit dans les rapports collectifs du travail. Par Marie-Louis Beaulieu. Québec: Les Presses Universitaires Laval, 1955. 540pp. En vente chez l'auteur, 945 avenue Bégin, Québec.] Relations industrielles / Industrial Relations, 13(3), 350-351. https://doi.org/10.7202/1022432ar

Tous droits réservés (C Département des relations industrielles de l’Université Laval, 1958
Ce document est protégé par la loi sur le droit d'auteur. L'utilisation des services d'Érudit (y compris la reproduction) est assujettie à sa politique d'utilisation que vous pouvez consulter en ligne.

https://apropos.erudit.org/fr/usagers/politique-dutilisation/ 


\section{RECENSIONS - BOOK REVIEWS}

Les conflits de droit dans les rapports collectifs $d u$ travail. Par MarieLouis Beaulieu. Québec: Les Presses Universitaires Laval, $1955.540 \mathrm{pp}$. En vente chez l'auteur, 945 avenue Bégin, Québec.

Les bons livres sont comme les bons vins: ils gagnent en saveur avec le passage des ans. L'ouvrage à la fois savant et pratique de Me Marie-Louis Beaulieu sur Les conflits de droit dans les rapports collectifs du travail est de ceux-là. Publié il y a trois ans, il a fait ses preuves aussi bien aux yeux du juriste le plus exigeant qu'à ceux du praticien des relations patronales-ouvrières assez patient pour en assimiler lentement le contenu. C'est d'ailleurs ce qui permet à oette recension de porter à coup sûr.

L'auteur est depuis plusieurs années titulaire de la chaire de Législation du travail et de sécurité sociale à la Faculté de droit de l'Université Laval, et chargé d'un cours sur les mêmes matières à la Faculté des sciences socia!es de la même université. En cette qualité, il a formé plusieurs générations d'étudiants aux réalités juridiques du drame des relations entre syndicat et direction chez nous. Et son volume est l'heureuse synthèse de son enseignement.

Me Beaulieu a voulu mettre en lumière toutes les lois qui régissent, au Québec, les relations entre patrons et ouvriers pris comme entités collectives. Pionnier en ce domaine, il a parfaitement réussi à en indiquer les sources juridiques, la structure, les détails et, le cas échéant, les défauts avec leurs solutions pertinentes.

Mais l'auteur a voulu donner plus de perspective et un meilleur éclairage à notre législation du travail en présentarnt, au début de son ouvrage, l'historique et le contenu de législations étrangères dont ici et là elle s'inspire. C'est ainsi que les cent premières pages de ce volume analysent succinctement, mais fort à propos, la législation du travail de la France (qui est sans contredit l'objet de prédilection de l'auteur), de l'Angleterre, des Etats-Unis et du. gouvernement fédéral canadien. Les. propos juridiques s'y trouvent encadrés. par des détails utiles sur le développement parallèle du syndicalisme dans les. divers pays.

Ainsi étayé, l'auteur s'engage avecpatience et compétence dans le dédale de nos lois québecoises de règlement des conflits de droit. Il passe tour à. tour en revue la Loi des différends. ouvriers de Québec, la Loi des syndicats professionnels, la Loi de la convention collective, la Loi du salaire minimum, la Loi des relations ouvrières, la Loi des différends entre les services publics et leurs salariés, la Loi concernant les corporations municipales et: scolaires et leurs salariés, etc. Chaqueloi est l'objet d'une étude détaillée: historique, objet, application et vue d'ensemble. Et cette série d'analyses. fouillées est suivie de deux chapitres: sur «la contrainte légale et l'intervention de l'Etat dans notre droit du travail » et une «vue d'ensemble de nos lois provinciales sur la réglementation du contrat de travail ».

Cette longue partie peut paraître fastidieuse au premier abord pour le profane; elle n'en est pas moins la. plus utile peut-être, et c'est certainement celle où l'auteur, excellent juriste, se sent le plus à l'aise. On y trouve' des notes précieuses sur les relations qui existent entre certaines lois (comme par exemple la Loi des relations ouvrières et la Loi des différends ouvriers de Québec), sur le sens du mot «différend », le caractère et le rôle précis des membres d'un conseil d'《arbitrange », l'équité et la bonne conscience comme guides dans le silence ou l'obscurité de la loi, l'extension de la convention collective par décret en regard de la convention collective particulière, la définition du «salarié », et ainsi de suite. Et les nombreuses notes historiques et junisprudentielles qui étoffent ces divers chapitres donnent à leur ensemble beaucoup de consistance. 
Le «Livre deuxième» est un bel effort de synthèse des lois précédemment analysées et de philosophie du droit. Il porte sur les rapports collectifs et les conflits de droit avec l'arrière-plan du social et de l'économique; il distingue conflit de droit et conflit d'intérêts; il établit enfin la relation entre ces notions juridiques et notre droit québecois du travail. On sait que la distinction formelle entre conflit de droit et conflit d'intérêts, si clairement posée en France et dans la plupart des pays du continent européen, n'apparaît pas dans notre droit.

L'auteur a un bon chapitre sur le recours au pouvoir judiciaire (e.g., par le bref d'injonction) dans les conflits de travail, sur les conflits qui malheureusement continuent à se livrer autour de la reconnaissance syndicale (conflits de droit, depuis la Loi des relations ouvrières de 1944) et sur le rôle, en cette matière, de la Commission de relations ouvrières.

Me Beaulieu, tout en distinguant les conflits de négociation et les conflits d'application de conventions collectives, prend bien soin de souligner un fait très important, à savoir que les premiers (conflits d'intérêts) aussi bien que les seconds (conflits de droit) peuvent naître après que la convention a été signée, si la convention et la loi sont muettes sur le problème gui se pose soudain pour les parties. Il introduit cenendant ici une troisième catégorie («mixte 》) de conflits qui est superflue. et qui pourrait faire naître de la confusion dans un domaine qui en a déjà sa bonne part.

Les notes de l'auteur sur l'«arbitrabilité » des griefs (les objections à l'arbitrage), la procédure arbitrale, les arbitres et le contrôle iudiciaire (par href de prohibition) et la sécurité syndicale viennent couronner un remarquable ouvrage.

On pourrait reprocher à l'auteur certains défauts de forme, comme parfois des longueurs non nécessaires et certaines répétitions (par exemple, il est question à trois endroits distinots du sens du mot «différend 》) dues peutêtre à la structure même du volume. Sans compter que la langue du juriste r'est pas le véhicule ordinaire de la pensée pour les praticiens des relations industrielles.

Mais tel quel, l'ouvrage de $\mathrm{Me}$ Beaulieu est une contribution de grande classe à un domaine du droit encore à se construire et que peu d'auteurs, chez ncus du moins, ont pu ou voulu aborder ḋe front. La qualité de ce livre nous invite à insister pour que son auteur continue à produire, pour le plus grand bien de la législation du travail chez nous, d'autres oeuvres marquées au coin d'un sens critique encore plus développé si possible.

Roger Chartier

\section{L'évolution du travail ouvrier aux usines} Renault. Par Alain Touraine. Paris: Editions du Centre national de la recherche scientifique, 1955 . 191pp.

Cette monographie est plus qu'une étude empirique ordinaire. Elle remet littéralement en question les perspectives les mieux acquises de la sociologie industrielle.

Pour en faire apparaître toute la valeur de nouveauté, c'est à l'enquête classique dirigée par Mayo à la Western Electric qu'il faudrait la comparer: au risque d'écraser $\mathbf{M}$. Touraine sous la comparaison, lui qui fut très loin de disposer des moyens mis en oeuvre dans la oélèbre monographie américaine. Celle-ci était axée - pour résumer grossièrement - sur l'étude des relations sociales à l'intérieur de l'usine, plus particulièrement sur le jeu des groupes spontanés. Elle nous a révélé ainsi dans l'entreprise une structure sociale dont l'image ne coincide pas avec les organigrammes ni, plus généralement, avec les définitions officielles $\mathrm{du}$ leadership formel. On peut dire que la conception courante de la sociologie industrielle date de cette prise de conscience. On en prendra une vue assez organique en paroburant, par exemple, le traité très répandu de Miller et Form. La sociologie industrielle y est définie par l'étude des groupes de travail et des relations de travail, du rôle joué par les travailleurs dans les grounes de travail, de l'organisation sociale de l'usine. Certains auteurs (européens surtout) ont formulé certaines critiques décisives à l'encontre de cette position du problème: elles se résu- 